\title{
Antioxidant cosmetotextiles: Cotton coating with nanoparticles containing vitamin $\mathrm{E}$
}

\author{
Fatemeh Shahmoradi Ghaheh ${ }^{\mathrm{a}}$, Akbar Khoddami ${ }^{\mathrm{a}}$, Farzaneh Alihosseini ${ }^{\mathrm{a}}$, Su Jing ${ }^{\mathrm{b}, \mathrm{c}}$, \\ Artur Ribeiro $^{\mathrm{b}}$, Artur Cavaco-Paulo ${ }^{\mathrm{b}, \mathrm{c}, *}$, Carla Silva ${ }^{\mathrm{b}, *}$ \\ a Department of Textile Engineering, Isfahan University of Technology, Isfahan, 84156-83111, Iran \\ b Centre of Biological Engineering, University of Minho, Campus of Gualtar, 4710-057, Braga, Portugal \\ c Jiangsu Engineering Technology Research Center for Functional Textiles, Jiangnan University, Wuxi, 214122, China
}

\section{A R T I C L E I N F O}

\section{Keywords:}

Cotton

Nanoparticles

a-tocopherol

Antioxidant activity

Release

Crock meter

\begin{abstract}
A B S T R A C T
In the present study, we coated cotton fabrics with protein-based nanoparticles containing vitamin $\mathrm{E}$ ( $\alpha$ tocopherol) by the pad-cure method. Scanning electron microscopy, Fourier transform infra-red spectroscopy, and air permeability analysis of coated samples confirmed the fixation of the nanoparticles onto the fabric's surface. The antioxidant activity of the coated fabrics was evaluated by 2,2'-Azino-bis(3-ethylbenzothiazoline-6sulfonic acid) (ABTS) free radicals reduction. Samples coated with nanoparticles containing the highest amount of encapsulated vitamin E ( $20 \%$ of the oil phase) showed the highest antioxidant activity. The protein-based coating was maintained for at least 10 washing cycles, demonstrating the reliability of the pad-cure method for the fixation of nanoparticles onto cotton surfaces. A methodology for nanoparticles release from the coated surfaces and their transfer to other substrates was demonstrated by the simple crock meter rubbing in the presence of sweat and protease. A high amount of material can be transferred and released to other substrates, such as textiles and skin, through the synergistic effect of sweat/protease and abrasion. An array of cosmetic and medical applications are possible with the developed coating and release methodology in which vitamin $\mathrm{E}$ would impart vital benefits as skin protection, anti-ageing product, or skin moisturizer.
\end{abstract}

\section{Introduction}

The use of textiles is common and transversal to all humans. Several types of textiles are used in different situations in life; we all use textiles, both during day life (clothing) and during the night (bed linens). These textiles are designed to have specific functions, namely protection, warmth, and support. Innovative methodologies have been established for the development of biological textiles with extra properties including breathability; texture; antimicrobial, and cosmetic properties [1-3]. Cosmetotextiles are examples of high-performance textiles representing a fusion of fabric materials with cosmetic active substances [4]. The cosmetic substances are attached to the fabrics, and when they come in contact with the human body and skin, they are transferred from the textile to the skin to reach the specific target [5]. Despite not being accepted as cosmetic products, they are able to impart skincare benefits, fighting ageing, and promote wellness and pleasant feelings $[1,6,7]$. Current cosmetotextiles in the market claim to be moisturizing, cellulite reducing, perfumed, body slimming, energizing, rejuvenating, refreshing, improving the firmness and elasticity of skin, or reducing the appearance of fine lines and wrinkles $[5,8]$.

Vitamin E (also known as $\alpha$-tocopherol) is a very well-known antioxidant agent, recognized to shut out hazardous oxygen and applied on textiles for anti-ageing purposes [6,7]. Microencapsulated vitamin E significantly increases the skin moisture and elasticity, thereby reducing the skin wrinkles and roughness. Despite having numerous advantages, its direct application into textiles is hindered by its low stability to heat and oxygen [2,9]. Microencapsulation has been devoted as the main technique for the incorporation of active substances into the textiles. It can be achieved by an array of methods in which the release of the active ingredient from the microcapsules occurs following heat, biodegradation, friction, or pressure between the body and fabric during use, breaking the capsules into fragments and releasing the encapsulated active ingredients $[10,11]$. Liposomes and polymeric and protein-based nanodevices have been successfully applied as vehicles to entrap and deliver vitamin E [10-13]. However, vitamin $\mathrm{E}$ incorporation into the lipid bilayer of liposomes or inside nanoparticles core is hindered by its lipophilicity and high molecular mass. We recently demonstrated that protein-based nanoemulsions can

\footnotetext{
* Corresponding authors.

E-mail address: carla.silva@ceb.uminho.pt (C. Silva).
} 
be successfully used for the encapsulation of this lipophilic product [14]. Protein nanoemulsions are dispersed in an aqueous medium where the protein remains at the interface covering the oil containing $\alpha$-tocopherol. The lipophilic nature of $\alpha$-tocopherol ensures that all the initial content introduced is totally encapsulated [14].

The main goal of this work was to develop cosmetotextiles with high-performance properties, namely antioxidant and skin moisture properties. The innovation behind this research is the development of a methodology that allows predicting further transfer behavior of the active substances to other substrates such as textiles and skin. Proteinbased nanoparticles composed of bovine serum albumin (BSA)/silk fibroin (SF) were previously developed and produced by oil-in-water methodology. Knitted cotton fabrics were coated with the previously developed oil-in-water nanoparticles containing vitamin $\mathrm{E}$ by the padcure method. The effectiveness and durability of the antioxidant properties were evaluated by scanning electron microscopy (SEM) and Fourier transform infra-red spectroscopy (FTIR) to confirm the presence and morphology of the nanoparticles.

The durability and transfer ability of the nanoparticles to other supports were assayed by washing and rubbing fastness, respectively. The antioxidant efficiency of vitamin E of coated samples was evaluated by the ABTS-scavenging methodology.

\section{Materials and methods}

\subsection{Materials and equipment}

Unless otherwise stated, all the solvents and reagents used in this study were commercially supplied by Sigma-Aldrich and used as received. Cocoons were donated by Dr. Silvia Cappellozza from 'Sezione Specializzata per la Bachicoltura' (Padova).

The experimental setup used for nanoparticles production was composed of a probe-type ultrasound source $(20 \mathrm{kHz}$ Sonics \& Materials Vibracell CV 33) fitted with a 3-mm diameter titanium micro-tip. Power delivery was controlled as percentage amplitude (40\%). The reaction vessel was an open glass cell (diameter $19 \mathrm{~mm}$ and height $75 \mathrm{~mm}$ ) containing the sample solution (16 mL). The sonochemical reactor temperature was controlled through a thermostated water bath with a freezer exchanger place within a thermojacket cell, which gave a steady operating temperature $\left(10 \pm 1{ }^{\circ} \mathrm{C}\right)$.

Bleached cotton fabrics used for the experiments had the following characteristics: rib structure (70 cotton/30 lycra); (12 wales $\times 19$ course) $/ \mathrm{cm} ; 500 \mathrm{~g} / \mathrm{m}^{2}$.

\subsection{Nanoparticles production and characterization}

The protein-based nanoparticles were prepared and characterized according to the methodologies previously described by Shahmoradi et al. (2016) [14].

\subsection{Washing of cotton samples prior to functionalization}

Prior to functionalization, cotton samples were washed with $1.25 \mathrm{~g} /$ L Lutensol AT 25 (non-ionic wetting agent from BASF) using a liquor ratio of $1: 7$ at $40{ }^{\circ} \mathrm{C}$ for $60 \mathrm{~min}$ in a rotawash machine at $20 \mathrm{rpm}$. The samples were then washed several times with tap water, followed by washing with distilled cold water. Finally, the samples were dried at $50{ }^{\circ} \mathrm{C}$.

\subsection{Coating of cotton fabrics with nanoparticles containing tocopherol - pad curing}

Cotton fabrics were coated with the produced nanoparticles using the pad-cure method. The samples were padded with water until the pick-up reached $70 \%$, followed by 20 passages with all produced nanoparticle solutions at $3.5 \mathrm{bar}$ and $3.02 \mathrm{~m} / \mathrm{min}$ of roll speed. Then, one set of samples was heat-cured at $50{ }^{\circ} \mathrm{C}$ for $20 \mathrm{~min}$ and the other set at $100{ }^{\circ} \mathrm{C}$ for $10 \mathrm{~min}$. The nanoparticle coating was verified by incubating the samples with Coomassie Brilliant Blue R-250 (0.5 g/L) at $60{ }^{\circ} \mathrm{C}$ for $60 \mathrm{~min}$.

\subsection{Scanning electron microscopy}

The morphology of functionalized cotton surface with nanoparticles was evaluated by SEM analysis. The grafted fabrics were scanned at different places on the sample using a NOVA Nano SEM 200 FEI instrument at 5000 and 50,000 $\times$ magnifications. The samples were previously freeze-dried and coated with a thick layer of gold before scanning with SEM, and all the images were carefully captured using the same sample position of wales and courses.

\subsection{Fastness to domestic washing}

The washing durability of functionalized samples was evaluated according to the AATCC test method 61(2A)-1996. In this approach, each cycle of washing process was performed with $2 \mathrm{~g} / \mathrm{L}$ non-ionic detergent for $45 \mathrm{~min}$ at $50{ }^{\circ} \mathrm{C}$ and $40 \mathrm{rpm}$ [15]. The washed fabric was dried at $50{ }^{\circ} \mathrm{C}$ for $30 \mathrm{~min}$ after each cycle of washing. The weight loss of the washed fabrics was analyzed after 10 washing cycles. Afterward, the samples were dyed with Coomassie Brilliant Blue, and $\Delta \mathrm{E}^{*}$ was evaluated by comparing the samples before and after washing.

\subsection{Washing fastness to sweat}

The samples' fastness to sweat was determined using ISO 105-E04 standard method using alkaline and acidic solutions. The test was performed at $37 \pm 2{ }^{\circ} \mathrm{C}$ for $4 \mathrm{~h}$ under a pressure of $5 \mathrm{~kg}$. Color fastness was further evaluated spectrophotometrically after dyeing with Coomassie Brilliant Blue because the coating is white and no color differences can be detected using levels $1-5$ of the standard procedure.

\subsection{Rubbing fastness to sweat and protease}

Functionalized samples were analyzed in terms of fastness to rubbing in the presence of alkaline and acidic sweat. Protease from Bacillus subtilis was also used to impregnate the testimony to evaluate its ability to hydrolyze the protein nanoparticles at the functionalized surfaces.

The experiments were conducted following the ISO 105-X12: 2001 standard method using a rubbing cotton reference impregnated with sweat or protease solution at a $100 \%$ pick-up.

\subsection{Air permeability}

Air permeability of the grafted cotton samples was performed using ASTM D737-96 Standard Test Method for Air Permeability of Textile Fabrics.

\subsection{Fourier transform infrared spectroscopy}

The chemical structure of BC samples was analyzed before and after swelling process and after PEG-FITC entrapment by FT-IR using an IR spectrophotometer (Nicolet Instrument Corporation, Waltham, MA, USA). Scans were completed between 4000 and $450 \mathrm{~cm}^{-1}$ at a resolution of $4 \mathrm{~cm}^{-1}$. Baselines for each sample spectrum were normalized using spectrum software.

\subsection{Moisture regain}

The moisture regain of non-coated and coated samples was determined according to ASTM D2495-07 (2012). The fabric samples were placed at room temperature for $24 \mathrm{~h}$ and then weighed $(\mathrm{w})$ and placed 
in an oven at $105^{\circ} \mathrm{C}$ for $1 \mathrm{~h}$. After exposure to $105^{\circ} \mathrm{C}$, samples were reweighed (d). The moisture regain of the samples was calculated according to Eq. (1):

Moistureregain $(\%)=\frac{\text { wetsampleweight }(w)}{\text { drysampleweight }(d)} \times 100$

\subsection{Release profile of $\alpha$-tocopherol}

The grafted samples of size $2 \times 2 \mathrm{~cm}$ were incubated with $95 \%$ ethanol under agitation (20 rpm) for $72 \mathrm{~h}$, and the release profile of $\alpha$ tocopherol was evaluated along time in ethanolic conditions. A calibration curve with several concentrations of tocopherol $(0.0005$, $0.001,0.0025,0.005,0.01$ and $0.02 \mathrm{mg} / \mathrm{mL}$ ) was performed in the same conditions.

\subsection{ABTS. ${ }^{+}$-scavenging activity of samples coated with $\alpha$-tocopherol- containing nanoparticles}

ABTS radical cation (ABTS. ${ }^{+}$)-scavenging activity was determined according to the method described by Re et al. (1999) [16] with some modifications. ABTS. ${ }^{+}$was produced by reacting $38.41 \mathrm{mg}$ ABTS (at a final concentration of $7 \mathrm{mM}$ ) and $6.623 \mathrm{mg}$ potassium persulfate (at a final concentration of $2.45 \mathrm{mM}$ ) in $10 \mathrm{~mL}$ demineralized water in an Erlenmeyer flask and maintaining the mixture in the dark at $26 \pm 3{ }^{\circ} \mathrm{C}$ for $12-16 \mathrm{~h}$ with magnetic stirring. An aliquot of blue-green ABTS. ${ }^{+}$ solution was then diluted with $95 \%$ ethanol to give an absorbance of $0.45 \pm 0.01$ at $734 \mathrm{~nm}$. For this, ABTS. ${ }^{+}$-adjusted solution $(2.5 \mu \mathrm{L})$ and ethanol $(197.5 \mu \mathrm{L})$ were mixed in an Erlenmeyer flask, and the absorbance at $734 \mathrm{~nm}$ corresponding to the blank control (E1) was recorded (Spectronic Genesys 5, Germany). For each sample test, Erlenmeyer flask containing the ABTS solution and the coated samples with different nanoparticle formulations were incubated. The absorbance of the reaction mixture was measured at each time point (E2). A control, i.e., sample without coating, was also tested to subtract the amount of ABTS that is adsorbed on the fabric. The ABTS. ${ }^{+}$scavenge $\%$ was calculated as follows:

ABTS..$^{+}$scavenge $(\%)=\left(\frac{E 1-E 2}{E 1}\right) \times 100$

\section{Results and discussion}

We successfully developed a method for the encapsulation and controlled release of vitamin E using protein nanoparticles. Luo et al. showed that chitosan nanoparticles coated with zein are a promising encapsulation and delivery system [17]. Melamine-formaldehyde microcapsules containing vitamin E were fixed onto dyed cotton fabrics and their durability was evaluated [2]. Poly(D,L-lactic-co-glycolide) nanoparticles with entrapped $\alpha$-tocopherol were synthesized by Zigoneanau et al. using surfactants and the release profile was evaluated [11]. Although promising results have been obtained by all these authors, some concerns related to nanoparticles' complexity, cytotoxi- city, and release profile have been considered. Moreover, their effective application onto surfaces such as that of textiles was poorly explored and characterized.

Our previous achievements enabled the efficient production and development of protein-based nanoparticles with entrapped vitamin $\mathrm{E}$ [14]. We study herein the application of these nanoparticles for the coating of cotton surfaces to impart them with antioxidant properties.

\subsection{Nanoparticles production and characterization}

Protein-based nanoparticles containing vitamin E were previously developed and optimized [14]. Nanoformulations of BS) and SF with entrapped $\alpha$-tocopherol (vitamin E) were produced through ultrasonic emulsification. Monodisperse populations with a particle size of $100-200 \mathrm{~nm}$ and highly negatively charged were obtained for all the tested formulations. Entrapment efficiencies of around 99\% revealed the effective encapsulation of $\alpha$-tocopherol into the produced nanoformulations. In general, these nanodevices did not induce significant cytotoxicity to human skin keratinocytes at all the concentrations tested [14].

\subsection{Coating of cotton fabrics with protein-based nanoparticles}

\subsubsection{Coating characterization}

The results related with the coating retention, presented in Table S.1 of support information, evidence the ability of the developed proteinbased nanoparticles to coat the fabric samples. The spectral values after coating and dyeing with Coomassie Brilliant Blue are higher for the coated samples when than for the uncoated ones. The nanoparticles retention reached values around 4-5\% (o.w.f), which was expected as all the coated samples were in the same state. These results also confirm the reproducibility of the coating methodology. This methodology was based on the pad curing of the samples, and the influence of the curing temperature was evaluated $\left(50{ }^{\circ} \mathrm{C}\right.$ and $\left.100{ }^{\circ} \mathrm{C}\right)$. A slight difference of spectral values $(K / S)$ is observed when tested with both temperatures. The curing at higher temperatures can contribute to the yellowing of the samples', therefore affecting the posterior coloration. The time of curing for $100{ }^{\circ} \mathrm{C}$ was therefore decreased to $10 \mathrm{~min}$ to avoid the degradation of the nanoparticles and decrease the yellowing of samples. Cotton can tolerate high temperatures for drying and curing; however, serious damage to the fibers can occur, causing changes in its morphology leading to breakage [18]. The fixation of different compounds is favored by high temperatures by improving a fiber's opening and a higher material deposition at the fabrics surface, however, compromising fiber's integrity.

Fig. 1 shows SEM photographs after coating of knitted cotton fabrics with nanoparticles containing vitamin $\mathrm{E}$. It can be observed that the spherical-shaped nanoparticles are located at the surface of the fibers and/or at the interstices between fibers. The interaction between cotton fiber surface and nanoparticles is mainly due to the intermolecular forces between hydroxyl groups of cotton and the amines of protein. This is also confirmed by the FITR spectra (Fig. 2), which evidences the $\mathrm{C}=\mathrm{O}$ stretching vibrations at $1742 \mathrm{~cm}^{-1}$ and the $\mathrm{C}=\mathrm{N}$ imine at
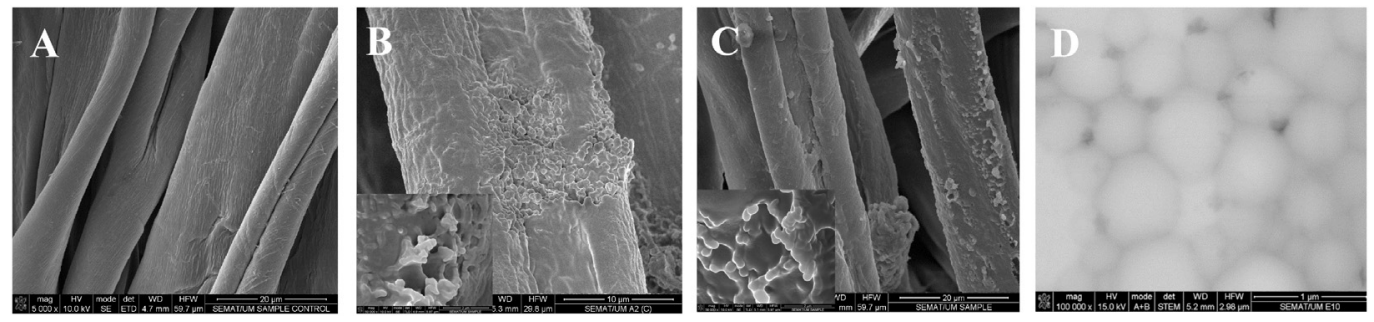

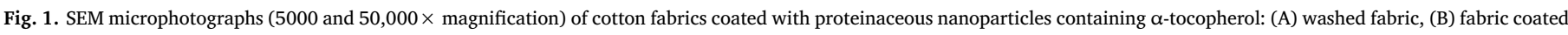

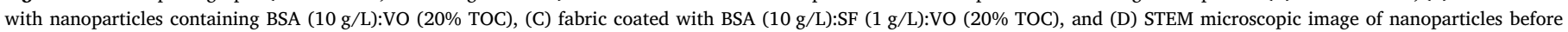
coating. 


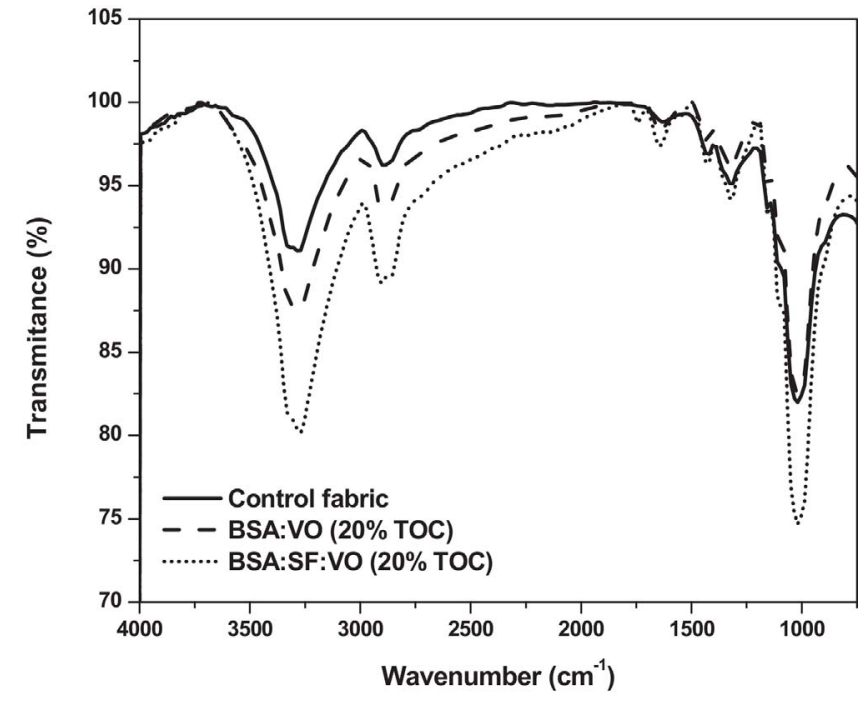

Fig. 2. FT-IR of coated cotton samples; (solid line) control washed fabric; (dashed line) cotton fabric coated with nanoparticles containing BSA:VO (20\% TOC); (short dot line) cotton fabric coated with nanoparticles containing BSA:SF:VO (20\% TOC).

$1645 \mathrm{~cm}^{-1}$ after the coating of cotton with the protein-based nanoparticles.

Moisture regain and air permeability are key comfort properties to consider in textile materials, especially when exposed to special finishing [19] (see Table S.2). After coating, it was observed that the fabrics were less permeable to air, with a decrease of around $40 \%$ in some samples. The coating with nanoparticles resulted in the filling of the gap between yarns and fibers hindering the airflow, resulting in a decrease of the air permeability values. The uniformity of nanoparticles coating was confirmed by this methodology as air permeability was performed at different points of the fabric, and all of them presented similar air permeability behavior. The pad-cure technique applied for fabric coating seems to be efficient in not only promoting a high level of material attachment but also ensuring the coating uniformity.

Moisture regain is defined as the percentage of water in a textile material after drying it in oven for a certain period. All fabric samples presented an increase in the moisture regain after nanoparticle coating (see Table S.2). This increase is due to the presence of protein-based nanoparticles containing hydrophilic groups at the surface of the fabrics.

\subsubsection{Coating durability and transfer to cotton substrates}

Several products in the market claim to impart additional properties to textile materials; however, some of them fail in the durability and effectiveness. Herein, in addition to coating of the textile fabrics with the produced nanoparticles, our goal was to test their effectiveness in terms of antioxidant behavior and the durability of the coating.

The fastness of the coated samples to sweat was assessed following
Table 2

Color difference of coated samples after washing fastness (ISO 105-C06: 2010) test and dyeing with $1 \mathrm{~g} / \mathrm{L}$ Coomassie Brilliant Blue R-250 for $1 \mathrm{~h}$ at $60{ }^{\circ} \mathrm{C}$.

\begin{tabular}{lll}
\hline & \multicolumn{2}{l}{$\Delta E$ (after 10 washing cycles) } \\
\cline { 2 - 3 } & Curing at $50{ }^{\circ} \mathrm{C}-20 \mathrm{~min}$. & Curing at $100{ }^{\circ} \mathrm{C}-10 \mathrm{~min}$. \\
\hline Non-coated fabric & 0.13 & 0.17 \\
BSA:VO (0\%TOC) & 3.55 & 2.55 \\
BSA:VO (10\%TOC) & 3.43 & 2.26 \\
BSA:VO (20\%TOC) & 3.84 & 2.48 \\
BSA:SF:VO (0\%TOC) & 3.27 & 2.88 \\
BSA:SF:VO (10\%TOC) & 3.63 & 2.53 \\
BSA:SF:VO (20\%TOC) & 2.78 & 2.61 \\
\hline
\end{tabular}

the standard methodology ISO 105-E04:2013. The results obtained (data not shown) revealed very good levels of fastness to sweat. However, these data can be misleading because the coating confers white color to the fabrics, and at the evaluation step of the multifiber, the color differences cannot be detectable.

\section{a) Fastness to sweat (acidic and alkaline) and to protease}

Considering this, the rubbing fastness of coated samples was assessed. After testing, the testimony samples were dyed with Coomassie Brilliant Blue and the spectral values were obtained (Tables 1 and Table S.3). The results evidence the transfer of the nanoparticles from the coated samples to the testimony (bleached cotton fabric) in the presence of acidic and alkaline sweat. The results obtained when the testimony was immersed first in protease solution show the hydrolytic effect of the enzyme, which degraded the proteinbased nanoparticles when in contact with the coated samples. A color decrease is hence observed. Fastness to rubbing tests evidence two main findings: the coated samples present low rubbing fastness in the presence of sweat together with the transfer of a considerable amount of coated material to cotton substrates. A simple abrasion is responsible for the transfer of the material that is more exposed and available for active compound release. Abrasion, sweat, and the presence of enzymes synergistically act on the release of the material from the coated surfaces. The release is of extreme importance from a practical point of view because a high level of release and transfer of the active substances from the coated material to the skin can be achieved.

\section{b) Fastness to domestic washing}

From the practical point of view, it is imperative that nanoparticles remain on a fabric's surface and be resistant to repeated washing. To investigate this, the coated samples were washed for 10 cycles and afterward colored with Coomassie Brilliant Blue. Color differences are presented in Table 2 (sample images at Table S.4). The results show $\Delta E$ of around 3.5 for samples cured at $50{ }^{\circ} \mathrm{C}$ and of 2.5 for samples cured at $100{ }^{\circ} \mathrm{C}$. This difference may be related to the use of a higher fixation temperature, which favors product attachment and thus hinders the washing out during the domestic washing process. The coating developed demonstrated a high washing resistance, showing acceptable durability.

Table 1

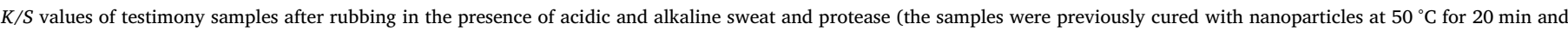
$100{ }^{\circ} \mathrm{C}$ for $10 \mathrm{~min}$ ) and dyed with $1 \mathrm{~g} / \mathrm{L}$ Coomassie Brilliant Blue R-250 for $1 \mathrm{~h}$ at $60^{\circ} \mathrm{C}$.

\begin{tabular}{|c|c|c|c|c|c|c|}
\hline \multirow[t]{2}{*}{ Samples } & \multicolumn{2}{|l|}{ Acidic sweat } & \multicolumn{2}{|l|}{ Alkaline sweat } & \multicolumn{2}{|l|}{ Protease } \\
\hline & $50{ }^{\circ} \mathrm{C}-20 \mathrm{~min}$ & $100^{\circ} \mathrm{C}-1 \mathrm{~min}$. & $50{ }^{\circ} \mathrm{C}-20 \mathrm{~min}$. & $100{ }^{\circ} \mathrm{C}-10 \mathrm{~min}$. & $50{ }^{\circ} \mathrm{C}-20 \mathrm{~min}$. & $100{ }^{\circ} \mathrm{C}-10 \mathrm{~min}$. \\
\hline Control & 0,47 & & 0,41 & & 0,60 & \\
\hline BSA:VO (0\% TOC) & 2.59 & 2.94 & 1.20 & 1.36 & 1.28 & 0.85 \\
\hline BSA:VO (10\% TOC) & 2.44 & 2.25 & 1.94 & 1.61 & 1.17 & 0.84 \\
\hline BSA:VO (20\% TOC) & 2.68 & 2.00 & 1.80 & 1.15 & 1.28 & 0.87 \\
\hline BSA:SF:VO (0\% TOC) & 1.29 & 1.87 & 1.56 & 1.03 & 1.12 & 0.91 \\
\hline BSA:SF:VO (0\% TOC) & 1.50 & 1.57 & 1.11 & 1.22 & 1.06 & 1.02 \\
\hline BSA:SF:VO (0\% TOC) & 1.80 & 1.60 & 1.97 & 1.5 & 1.11 & 0.87 \\
\hline
\end{tabular}


A)

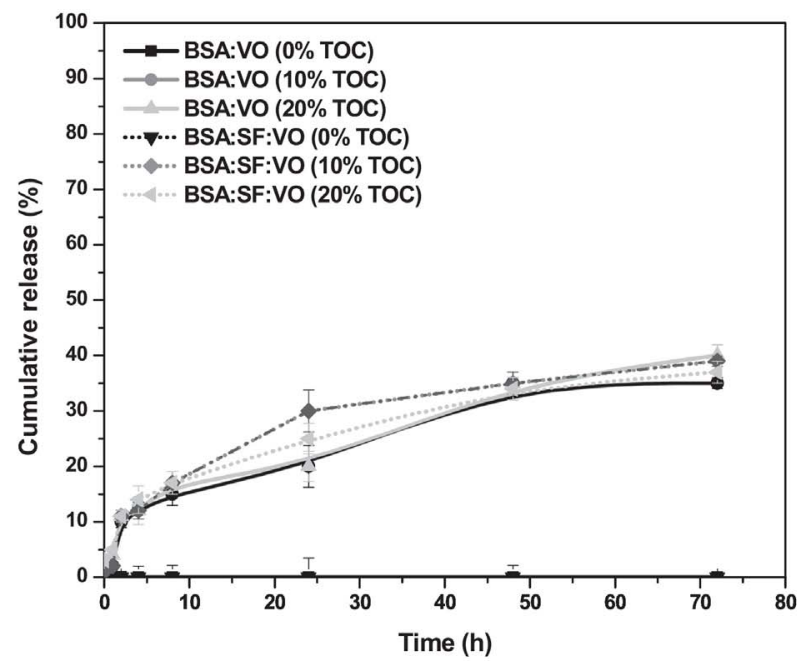

B)

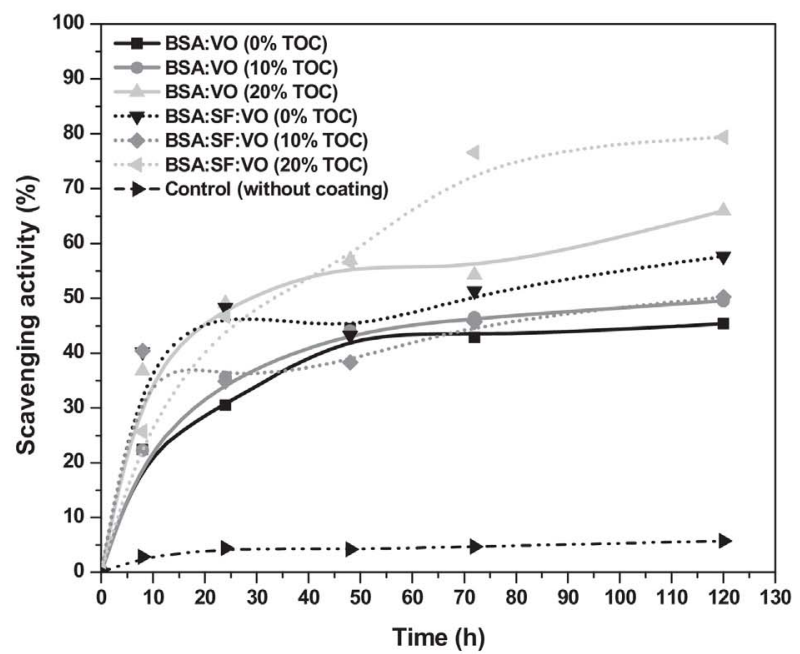

Fig. 3. (A) Release profile of $\alpha$-tocopherol from fabric-loaded nanoparticles in $95 \%$ ethanol at $37{ }^{\circ} \mathrm{C}$ and (B) antioxidant activity of coated fabrics in the presence of $\mathrm{ABTS}{ }^{+}$.

\subsection{Release and activity of $\alpha$-tocopherol from coated fabrics}

Because of the lipophilic nature of $\alpha$-tocopherol, its release profile from the coated cotton fabrics was studied in $95 \%$ ethanol solutions. The release profile shows an initial burst effect in the first few hours of incubation, and after $10 \mathrm{~h}$, almost $20 \%$ of release is evident. The maximum release obtained for coated samples previously washed was $40 \%$ of the initial loaded vitamin E. The release of active substances from nanodevices is usually dependent on several factors: the penetration of the release medium into the protein-based nanoparticles, the diffusion of the substance through the matrix, the degradation of the nanoparticle, and the release and transfer of the coated nanoparticles to the release medium [11]. The release profile achieved herein is the result of the cumulative effects of all these factors. The burst release can be attributed to the amount of $\alpha$-tocopherol adsorbed into the nanoparticles surface. Its affinity to the release medium can also contribute to its fast diffusion to the medium. However, the encapsulated vitamin E slowly diffuses to the exterior medium as it is entrapped into the protein/oil matrix.

The antioxidative capacity test was used to confirm the ability of protein-based nanoparticles containing $\alpha$-tocopherol to induce antioxidative properties to the cosmetotextiles. The coated fabrics were tested according to the international standard test ABTS method. This method measures the free radical reduction in the solution containing the functionalized cotton fabrics. Fig. 3B contains a graphical representation of the kinetic antioxidative properties for washed pre-treated samples. The lowest antioxidative capacity is shown for the reference fabric (without coating). As expected, the samples coated with the nanoparticles containing higher amount of tocopherol encapsulated presented the highest values of antioxidant activity.

\section{Conclusions}

This study explored the functionalization of cotton fabrics to impart them with antioxidant properties. Protein-based nanoparticles incorporating vitamin $\mathrm{E}$ ( $\alpha$-tocopherol) in their composition were efficiently coated onto cotton fabrics by the pad-cure methodology. Different techniques including SEM, FTIR, dyeing, and air permeability confirmed and validated the uniformity of the coating and the efficiency of the pad-cure method. The release profile of vitamin E showed an initial burst in the initial time points reaching the stationary level of around $40 \%$ after $48 \mathrm{~h}$ of incubation. The effectiveness of the antioxidant activity was evaluated by ABTS free radical reduction, and the results show that all the coated samples presented antioxidant activity when compared with the non-coated fabrics. The highest antioxidant values were observed for the samples coated with nanoparticles incorporating the highest amount of vitamin $\mathrm{E}$ at the oil phase.

Washing and rubbing fastness methods were conducted to evaluate coating durability, and data revealed the reliability of the pad-cure methodology for the coating of cotton surfaces. The release of the coating to other substrates was efficiently achieved by a simple rubbing test where a considerable amount of material was transferred to a cotton samples substrate. This technique evidenced the effect of both abrasion and sweat/or enzyme for the release of the active substances from the coated samples. For a practical application in cosmetotextiles, they will act synergistically, promoting the release and transfer of the material coated to the skin.

As a reliable and low-cost method, the pad curing of cotton fabrics with nanoparticles containing vitamin $\mathrm{E}$ can contribute to increase the cosmetic solutions available on the market.

\section{Acknowledgments}

This study was supported by the Portuguese Foundation for Science and Technology (FCT) under the scope of the strategic funding of UID/ BIO/04469/2013 unit and COMPETE 2020 (POCI-01-0145-FEDER006684) and BioTecNorte operation (NORTE-01-0145-FEDER000004) funded by the European Regional Development Fund under the scope of Norte2020 - Programa Operacional Regional do Norte. Artur Ribeiro thanks FCT for the SFRH $\backslash B P D \backslash 98388 \backslash 2013$ grant. Fatemeh Shahmoradi Ghaheh thanks the Iran Ministry of Science, Research and Technology for the monetary support.

\section{Appendix A. Supplementary data}

Supplementary data associated with this article can be found, in the online version, at http://dx.doi.org/10.1016/j.procbio.2017.04.020.

\section{References}

[1] C. Alonso, M. Martí, C. Barba, M. Lis, L. Rubio, L. Coderch, Skin penetration and antioxidant effect of cosmeto-textiles with gallic acid, J. Photochem. Photobiol. B 156 (2016) 50-55.

[2] K. Son, D.I. Yoo, Y. Shin, Fixation of vitamin E microcapsules on dyed cotton fabrics, Chem. Eng. J. 239 (2014) 284-289.

[3] O. Tzhayik, A. Lipovsky, A. Gedanken, Sonochemical fabrication of edible fragrant antimicrobial nano coating on textiles and polypropylene cups, Ultrason. Sonochem. (2017), http://dx.doi.org/10.1016/j.ultsonch.2016.08.020. 
[4] N. Azizi, Y. Chevalier, M. Majdoub, Isosorbide-based microcapsules for cosmetotextiles, Ind. Crops Prod. 52 (2014) 150-157.

[5] I. Holme, Innovative technologies for high performance textiles, Color. Technol. 123 (2) (2007) 59-73.

[6] R. BRIGELIUS-FLOHÉ, M.G. TRABER, Vitamin E: function and metabolism, FASEB J. 13 (10) (1999) 1145-1155.

[7] P.M. Bramley, I. Elmadfa, A. Kafatos, F.J. Kelly, Y. Manios, H.E. Roxborough, W. Schuch, P.J.A. Sheehy, K.H. Wagner, E. Vitamin, J. Sci. Food Agric. 80 (7) (2000) 913-938.

[8] C. Alonso, M. Martí, V. Martínez, L. Rubio, J.L. Parra, L. Coderch, Antioxidant cosmeto-textiles: skin assessment, Eur. J. Pharm. Biopharm. 84 (1) (2013) 192-199.

[9] M.J. Abla, A.K. Banga, Formulation of tocopherol nanocarriers and in vitro delivery into human skin, Int. J. Cosmet. Sci. 36 (3) (2014) 239-246.

[10] J. Hategekimana, K.G. Masamba, J. Ma, F. Zhong, Encapsulation of vitamin E: Effect of physicochemical properties of wall material on retention and stability, Carbohydr. Polym. 124 (2015) 172-179.

[11] Z. Imola Gabriela, A. Carlos Ernesto, S. Cristina Mirela, Nanoparticles with entrapped $\alpha$-tocopherol: synthesis, characterization, and controlled release, Nanotechnology 19 (10) (2008) 105606.

[12] Y. Yang, D.J. McClements, Encapsulation of vitamin E in edible emulsions fabricated using a natural surfactant, Food Hydrocolloids 30 (2) (2013) 712-720.
[13] A.H. Saberi, Y. Fang, D.J. McClements, Fabrication of vitamin E-enriched nanoemulsions: factors affecting particle size using spontaneous emulsification, J. Colloid Interface Sci. 391 (2013) 95-102.

[14] F.S. Ghaheh, A. Khoddami, F. Alihosseini, A. Gomes, A. Ribeiro, A. Cavaco-Paulo, C. Silva, Protein-based nanoformulations for $\alpha$-Tocopherol encapsulation, Eng. Life Sci. (2016), http://dx.doi.org/10.1002/elsc.201600188.

[15] R. Dastjerdi, M. Montazer, S. Shahsavan, A novel technique for producing durable multifunctional textiles using nanocomposite coating, Colloids Surf. B: Biointerfaces 81 (1) (2010) 32-41.

[16] R. Re, N. Pellegrini, A. Proteggente, A. Pannala, M. Yang, C. Rice-Evans, Antioxidant activity applying an improved ABTS radical cation decolorization assay, Free Radic. Biol. Med. 26 (9-10) (1999) 1231-1237.

[17] Y. Luo, B. Zhang, M. Whent, L. Yu, Q. Wang, Preparation and characterization of zein/chitosan complex for encapsulation of $\alpha$-tocopherol, and its in vitro controlled release study, Colloids Surf. B: Biointerfaces 85 (2) (2011) 145-152.

[18] Y.L. Buisson, K. Rajasekaran, A.D. French, D.C. Conrad, P.S. Roy, Qualitative and quantitative evaluation of cotton fabric damage by tumble drying, Text. Res. J. 70 (8) (2000) 739-743.

[19] A. Afzal, S. Ahmad, A. Rasheed, F. Ahmad, F. Iftikhar, Y. Nawab, Influence of fabric parameters on thermal comfort performance of double layer knitted interlock fabrics, Autex Res. J. (2016). 